University of Nebraska - Lincoln

DigitalCommons@University of Nebraska - Lincoln

Community and Regional Planning Program:

Faculty Scholarly and Creative Activity

Community and Regional Planning Program

$5-10-2020$

\title{
How cropland losses shaped by unbalanced urbanization process?
}

Bingwen Qiu

Fuzhou University, qiubingwen@fzu.edu.cn

Haiwen Li

Fuzhou University

Zhenghong Tang

University of Nebraska - Lincoln, ztang2@unl.edu

Chongcheng Chen

Fuzhou University

Joe Berry

Carnegie Institution for Science, Stanford, CA, jberry@carnegiescience.edu

Follow this and additional works at: https://digitalcommons.unl.edu/arch_crp_facultyschol

Part of the Urban, Community and Regional Planning Commons

Qiu, Bingwen; Li, Haiwen; Tang, Zhenghong; Chen, Chongcheng; and Berry, Joe, "How cropland losses shaped by unbalanced urbanization process?" (2020). Community and Regional Planning Program: Faculty Scholarly and Creative Activity. 36.

https://digitalcommons.unl.edu/arch_crp_facultyschol/36

This Article is brought to you for free and open access by the Community and Regional Planning Program at DigitalCommons@University of Nebraska - Lincoln. It has been accepted for inclusion in Community and Regional Planning Program: Faculty Scholarly and Creative Activity by an authorized administrator of DigitalCommons@University of Nebraska - Lincoln. 


\title{
How cropland losses shaped by unbalanced urbanization process?
}

\author{
Bingwen Qiu, ${ }^{1}$ Haiwen Li, ${ }^{1}$ Zhenghong Tang, ${ }^{2}$ \\ Chongcheng Chen, ${ }^{1} \&$ Joe Berry ${ }^{3}$
}

\footnotetext{
1 Key Laboratory of Spatial Data Mining \& Information Sharing of Ministry of Education, National Engineering Research Centre of Geospatial Information Technology, Fuzhou University, Fuzhou 350116, Fujian, China

2 Community and Regional Planning Program, University of Nebraska-Lincoln, Lincoln, Nebraska 68588, USA

3 Department of Global Ecology, Carnegie Institution for Science, Stanford, CA 94305, USA

Corresponding author - B. Qiu, Spatial Information Research Centre of Fujian Province, Yangguang Keji Building, floor 8th, Xueyuan Road 2, Fuzhou University, Fuzhou 350116, Fujian, China. Email qiubingwen@fzu.edu.cn
}

\begin{abstract}
Urbanization has widely known to directly consume swaths of cropland worldwide. Knowledge on what kinds of urbanization processes spared cropland is important for land use planning. This study offered insights on the impact of city level (city hierarchy: from the 1st to the 6th Tier cities) and urbanization modes (mega-city, city, town and village modes) on cropland losses through a first-ever continuous national survey on 345 prefectural level cities or above in mainland China from 2003 to 2016. We found that higher tier cities were associated with more direct and severe losses. Specifically, over $80 \%$ of the recent urbanization formed on cropland in the 1st Tier cities, and the newly 1st Tier cities suffered the most rigorous losses. At national level, mega-city mode urbanization resulted in direct cropland losses (80\%)
\end{abstract}

Published in Land Use Policy 96 (2020) 104715

DOI:10.1016/j.landusepol.2020.104715

Copyright (c) 2020 Elsevier Ltd. Used by permission.

Submitted 21 September 2019; revised 21 March 2020; accepted 25 April 2020; published 10 May 2020. 
and the village mode was associated with prominent high-quality ratio (45\%). Town mode spared cropland more than village mode. However, ranking with urbanization mode was less obvious and even changed in the lower-Tier cities. At national scale, around $1.45 \%$ of the total cropland area (approximately $2297 \mathrm{~km}^{2}$ per year), including $1.06 \%$ of high-quality cropland area (approximately $852 \mathrm{~km}^{2}$ per year), has been permanently lost. The most rapid cropland loss was in $2009\left(3464 \mathrm{~km}^{2}\right)$, and that of high-quality cropland occurring in $2007\left(1775 \mathrm{~km}^{2}\right)$. Over $95 \% \mathrm{crop}-$ land losses located in the east of the Hu line. Findings in this study called for target adaptive planning with full considerations of city hierarchy and urbanization mode. Particularly, land use policies to effective support land development in small towns can potentially relief pressure on cropland.

Keywords: Cropland losses, Unbalanced urbanization, City hierarchy, Coupling, China

\section{Introduction}

Urbanization is an irreversible trend worldwide (Liu, 2018) and always unavoidably occupies the world's most productive cropland (Seto and Ramankutty, 2016; Song et al., 2018; van Vliet, 2019). Competition for high-quality land is projected to continue and even accelerate, given that 6o\% global irrigated croplands are located near urban areas and an additional 70\% increase in agricultural production will be needed by 2050 (d'Amour et al., 2017). Under the context of fast economic growth since 1980s, China has undergone rapid urbanization largely at the expense of cropland loss (Kong, 2014; Zuo et al., 2018). The accelerating competition for good-quality land has exacerbated the challenge of feeding more than 1.4 billion people with limited good quality cropland (Chen, 2007; Song et al., 2015). Cropland loss has been a significant concern in terms of maintaining self-sufficiency in food production and increasing urbanization in China (Barthel et al., 2019; Deng et al., 2014).

Urbanization process is worldwide unbalanced (d'Amour et al., 2017; Cao et al., 2014). The unbalanced development could be caused by different physical locations, varied social-economic driving forces, and other contextual factors with references to the location theory and regional economic growth (North, 1955). China has long encouraged an unbalanced urbanization strategy since the economic reforms that began in the 1980s (Cao et al., 2014). The unbalanced development strategy could be reflected by the highly hierarchical city system 
(namely the Tiered city system) (Cao et al., 2014; Zhang et al., 2019). However, the unbalanced urbanization strategy was challenged for its sustainability (Cao et al., 2014; Liu, 2018; Long et al., 2010). Nowadays, the state of China calls for coordinated development and rural revitalization (Liu and Li, 2017). For instance, since the 11th five-year plan, the state introduced several regional plans target regional coordination (e.g., development of the west, revitalization of the northeast, and rise of the central) (Liu et al., 2014b).

Guiding urban development towards more sustainable trajectories is important (van Vliet, 2019). Although urban expansion brings unavoidable challenges for competitive land use, it also provide important opportunities to determine growth patterns of urbanization (van Vliet, 2019). Knowledge on how cropland loss shaped by unbalanced urbanization processes is important for land use planning. Recent studies have found that cropland has been significantly lost with uneven development patterns in different geographical regions (Liu et al., 2014a; van Vliet et al., 2017). Huge cropland losses occurred surrounding mega-cities (d'Amour et al., 2017). This dynamic patterns adds pressure to potentially strained future food systems and threatens livelihoods in vulnerable regions (Barthel et al., 2019; d'Amour et al., 2017). Nevertheless, some claimed that expansion of small towns/ villages or rural residential land experienced on overwhelming majority from cropland (Long et al., 2010, 2009; Skinner et al., 2001). Broader analysis of both rural and central areas is needed (Skog and Steinnes, 2016). A thorough investigation with consideration of city hierarchy and urbanization mode (mega-city, city, town and village modes) at large scale (i.e. regional, national or above) is required for deep understanding on how unbalanced urbanization processes shaped the cropland losses (Deng et al., 2015; Liu et al., 2014b; Zhang et al., 2019).

This study aims to address this requirement based on spatiotemporal explicit annual datasets during the past two decades. Specifically, we directly address the following scientific questions: 1 ) whether cropland loss was recently decelerated and decoupled from urban expansion? 2) How was cropland losses shaped by city hierarchy and urbanization mode in China? Particularly, this study examines whether the lower-Tier cities or village mode really experience less serious losses during the past two decades? 


\section{Materials and methods}

\subsection{Data sources}

\subsubsection{Annual cropland loss and urbanization datasets}

The distribution of cropland in 2000 was derived from the GlobeLand3o dataset (Chen et al., 2015) (www.globallandcover.com/ GLC30Download/index.aspx). A recent study proposed an automatic approach for continuously tracking landscape changes (Qiu et al., 2019), which provided the annual newly impervious surface areas, bare lands, and inundation areas in China from 2001 to 2017 (Qiu et al., 2019) (downloaded from https://drive.google.com/drive/folders/1 ZGSk3sPo40b77lmLTHo1Box1P). Cropland would be permanently lost through a conversion to newly impervious surface areas. This study focused on cropland loss due to urbanization. Therefore, datasets on cropland loss was derived based on the cropland distribution map in 2000 (Chen et al., 2015) and annual newly impervious surface areas in China from 2001 to 2017 (Qiu et al., 2019).

Urbanization is commonly defined as the process by which an area becomes more urban (Gaston, 2010), where urban here refers to 'impervious surface' (Chen et al., 2015). Annual newly impervious surface area (newly urban land) was also obtained based on a recent study for automatic tracking landscape changes (Qiu et al., 2019). Annual newly urban land from cropland was calculated in order to estimate cropland losses due to urban expansion (Fig. S1). The 30m cropland dataset was aggregated to 500m through a majority strategy in order to correspond to datasets of landscape changes from 2003 to 2016 (Qiu et al., 2019).

\subsubsection{Cropland quality/productivity datasets}

The gross primary production (GPP)/net primary production products have been applied to evaluate changes in agricultural productivity (He et al., 2017; Yan et al., 2009). A recent vegetation photosynthesis model based on gross primary production product was developed (Zhang et al., 2017). High-quality cropland was identified by the 8day composite 500m MODIS gross primary production products based on the averaged level of annual peak GPP (Fig. S1). Annual peak was applied in order to relieve influences by cropping intensity, crop phenology and data contamination (Qiu et al., 2016). 
a



b

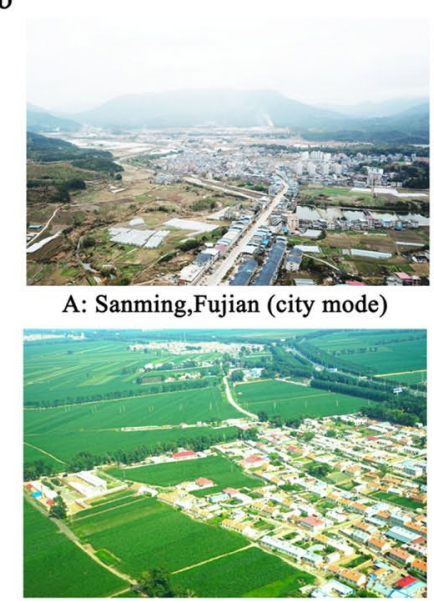

B: Panjin,Jilin (town mode)

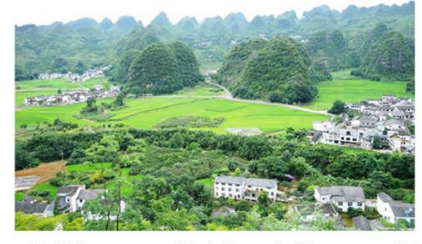

C: Xingyang, Guizhou (village mode)

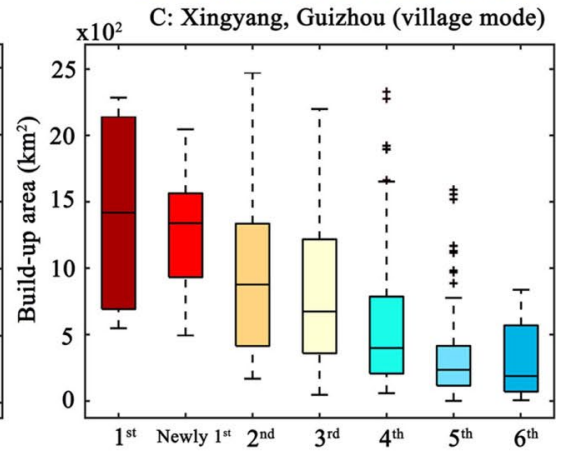

Fig. 1. Maps of different Tier cities (a), photos of cropland losses in different urbanization modes (b), boxplots of population, GDP and build-up areas among different Tier cities (c).

\subsubsection{Social-economic datasets of prefecture-level or above cities}

Data on sites of Chinese cities/towns were downloaded from http:// www.resdc.cn/. There were a total of 345 cities/towns at the prefecture-level or above in China (Fig. 1). A prefectural level city is often not a "city" in the usual sense of the term, but instead an administrative unit comprising, typically, a main central urban area, and its much larger surrounding rural area containing many smaller cities, towns and villages (https://en.wikipedia.org/wiki/Prefecture-level city). 
The term 'city' in this study refers to administrative prefecture-level city instead of a built-up city. Detailed datasets of the prefecture-level or above cities were provided in Table S1. The socio-economic datasets such as population and Gross Domestic Product (GDP) in Table S1 were downloaded from the National Bureau of Statistics of China (http://www.stats.gov.cn/english/). Datasets on cropland, cropland loss and built-up areas were aggregated based on the corresponding datasets in section 3.1.1.

Numerous Chinese cities have experienced fast and unbalanced development. The central government divided cities into different tiers for better management. This city hierarchy system takes into account of multiple indicators from business or internet data which can reflect the economic development (i.e. resource agglomeration, transportation pivotability, population activity, lifestyle diversity, and future plasticity) instead of simply by population size of urban extent (Ou et al., 2019). Cities at prefecture-level or above were sorted into six tiers according to the development level. According to the Chinese city hierarchy system, there are 4, 15, 30, 70, 90 and 128 cities listed on the 1st, newly 1st, 2nd, 3rd, 4th and 5th Tier orders, respectively (Fig. 1) (Table S1). Other prefecture-level cities (8 cities) were the 6th Tier cities. The Tier city system does not exactly correspond with the administration level. I.e. The newly 1st Tier cities include directly controlled municipalities, provincial capital cities as well as prefectural level cities. Higher Tier cities are typically associated with large population, build-up areas and higher GDP (Fig. 1, Fig. S2).

The unbalanced development strategy in China can be illustrated by the $\mathrm{Hu}$ line (also called the Heihe-Tengchong line) (Fig. 1), the east of the line accounted for $96 \%$ population with just over one third land (Bai et al., 2014). The unbalanced development strategy accelerated excessive growth of mega-cities (Cao et al., 2014; Zhang et al., 2019). Higher-level cities in eastern China received stronger policy supports from the central government and stimulate rapid economic development (Cao et al., 2014). A majority cities (277 out of 345, around $80 \%$ ) situated in the east of the Hu line (Fig. 1). All the 1st and newly 1st cities distributed in the east. Cities located in the west of the $\mathrm{Hu}$ line are lower-Tier cities, which include only one Tier-two city, four Tier three cities, four Tier-four cities, 55 Tier-five cities and 4 Tier-six cities. In the east, 36\% of national land support $96 \%$ of the population (Bai et al., 2014). There are 96\% national high-quality cropland, 
96\% national GDP and over 90\% national build-up area coexisted in the east (Figs. S1 and S2).

\subsection{Methodology}

The Loss Ratio in cropland (LR)/high-quality cropland (LRH) was designed to measure the proportion of cropland/high-quality cropland loss at a national scale or in each agricultural region/city/distance (Fig. 2). Contribution Rate (CR) was developed to assess the impact of urban expansion on cropland. High-Quality Ratio (HQR) was exploited to measure the influence of urban expansion on high-quality cropland. Changes in these indictors could reveal changes in the effectiveness of cropland protection policies at a national scale during the past two decades. Lower indicator values signify better protection of cropland quantity and productivity.

$$
\begin{array}{r}
C R=\frac{C L_{t}}{U L_{t}} \times 100 \% \\
H Q R=\frac{C L H_{t}}{C L_{t}} \times 100 \%
\end{array}
$$

where $U L_{t}$, indicates newly urban land at year t; $C L_{t}$ and $C L H_{t}$ represent the lost areas of cropland or high-quality cropland, respectively, due to urban expansion at year $t$.

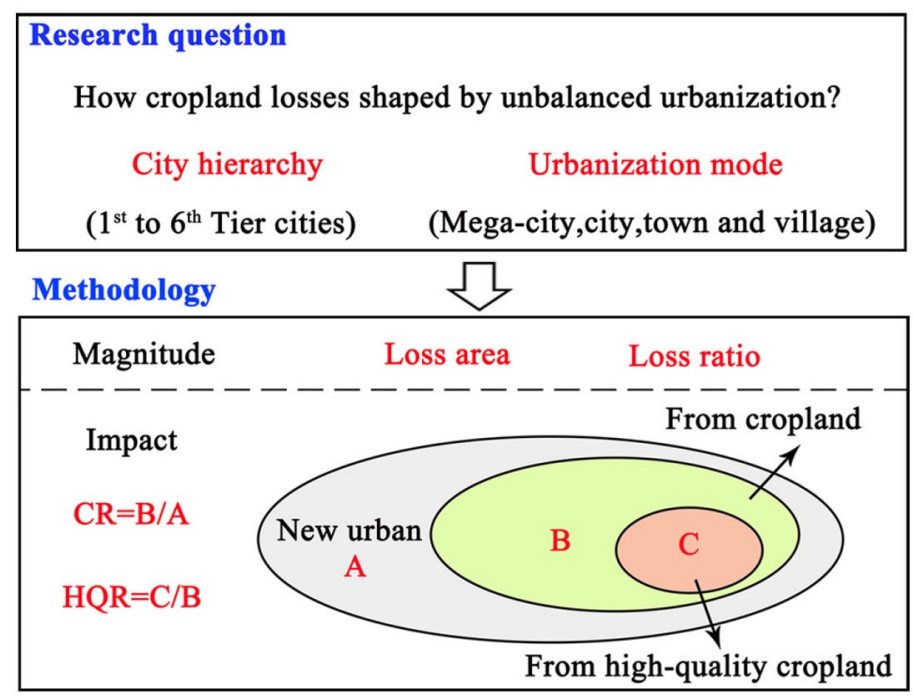

Fig. 2. The methodology framework. 
In order to evaluate the influences of different urbanization modes, we divided the patches into four categories based on the sizes of impervious surface area (Fig. 2, Fig. S1). The first category includes all patches with impervious surface area less than $0.5 \mathrm{~km}^{2}$, which are mostly equivalent to rural residential areas and are defined as the "village" mode (Deng et al., 2015). The second category, "town" mode urbanization, comprises all patches of impervious surface area within $0.5-5 \mathrm{~km}^{2}$, which corresponds to the size of a typical large township area. The third category, "city" mode urbanization, refers to impervious surface area larger than $5 \mathrm{~km}^{2}$ but less than 50 $\mathrm{km}^{2}$. And the forth category, "megacity" mode urbanization, is particularly designed to explore the patterns of large continuous urban settlement more than $50 \mathrm{~km}^{2}$. Distribution map of impervious surface area in 2016 was applied for this purpose, which was derived through merging newly impervious surface area from 2001 to 2016 (Qiu et al., 2019) and build-up area in 2000 (Liu et al., 2014a). Distances to the nearest cities/towns centers were calculated in order to explore the spatiotemporal patterns of cropland losses within urban, suburban and rural regions.

\section{Results}

\subsection{Overall cropland loss due to urbanization at a national scale}

At national level, there were a total of $32,153 \mathrm{~km}^{2}$ losses of cropland due to urban expansion during the study period (2003-2016) (Fig. 3, Table S2). Around 1.45\% of total cropland area was lost at a national scale, which contributed to $67.20 \%$ of newly developed urban lands. Over one-third loss (37.09\%) was of high-quality cropland. Urban expansion encroached $11,925 \mathrm{~km}^{2}$ high-quality cropland and around $1.06 \%$ of total high-quality cropland area was permanently lost.

Annual losses accelerated rapidly in the 2000 s and gradually declined in the 2010s (Fig. 3, Table S3). The speediest period of losses was exhibited in 2009 and $3464 \mathrm{~km}^{2}$ cropland was encroached by urban expansion in this single year. The fastest period of high-quality cropland losses occurred in 2007, with $1775 \mathrm{~km}^{2}$ losses in this single 
(a): Cropland losses

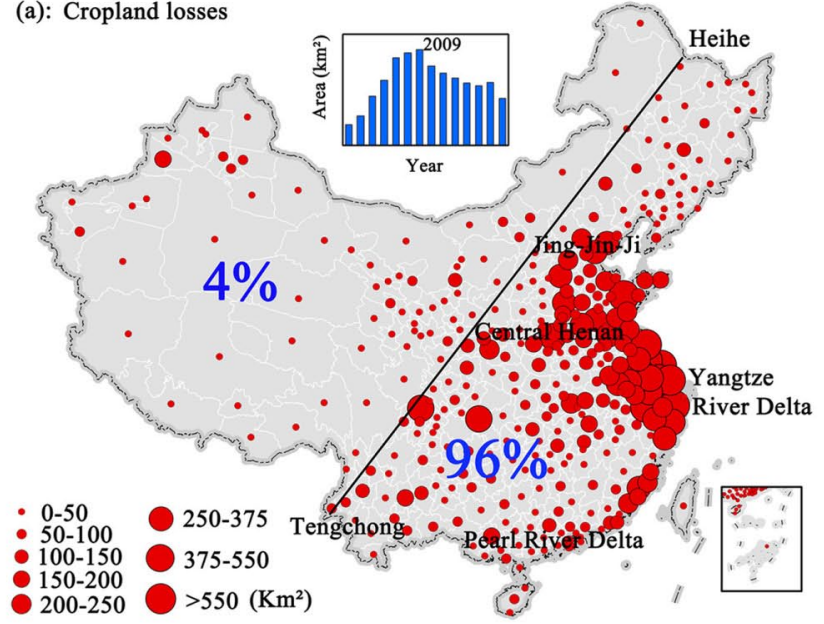

(c): $\mathrm{CR}$

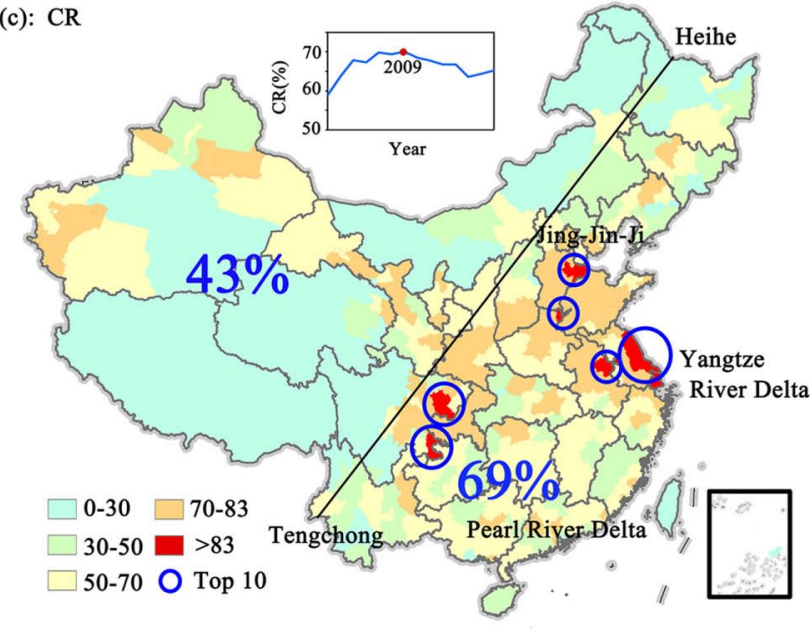

(b): High-quality losses

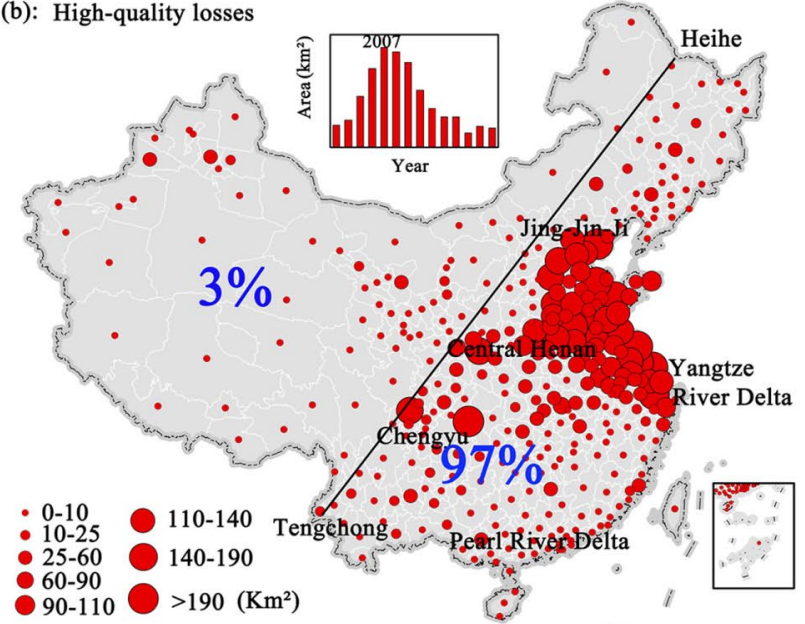

(d): HQR

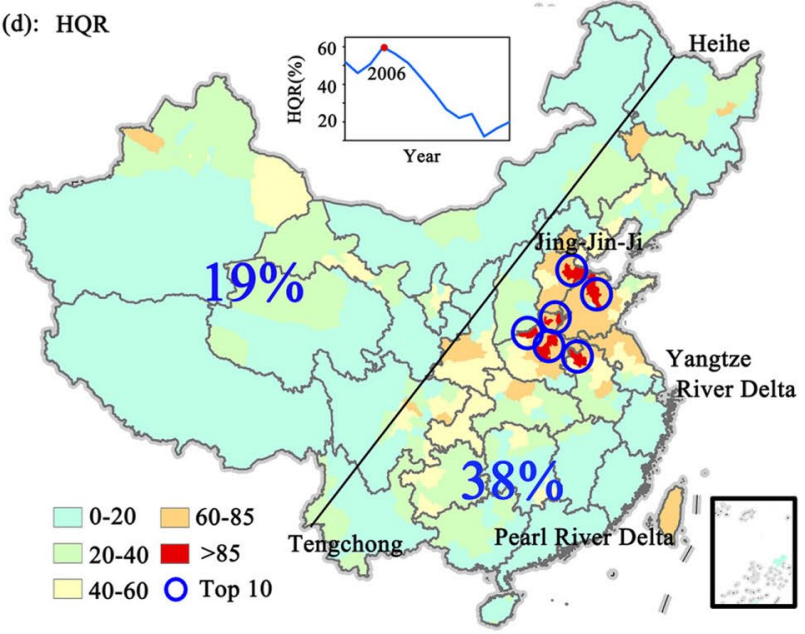

Fig. 3. Maps of cropland losses (a), high-quality cropland losses (b), contribution rate (CR) (c) and high-quality ratio (HQR) (d) in China during the period 20032016. Notes: The bar in Fig. 2 (a), (b), (c), (d) denoted its corresponding histogram/ line chart of annual national changes. The line plot is the Hu line and the percentage of losses at national scale (Fig. 2 (a),(b)) or averaged values (Fig. 2 (c),(d)) in the west and east side of the Hu line were provided. Top ten highest cities/towns were highlighted in Fig. 2 (c), (d).

year. Different than the distinctive annual variations of lost areas, the contribution rate was continuously high (60-70\%). However, the high-quality ratio of losses decreased sharply after reaching its peak in 2006 (around 6o\%), which achieved the lowest value in 2014 (12\%) and gradually increased afterwards (Fig. 3). 


\subsection{Impact from city level among the city hierarchy}

The magnitude of cropland losses was highly unbalanced among the city hierarchy. Cropland losses significantly increased from the lower-Tier cities to the higher-Tier cities (Table S2). In particularly, the loss ratio (LR) in the 1st Tier cities was over 4 (4.37) times of the national average, followed by the newly 1st and 2nd Tier cities (around 2.8 times of the national average). Loss ratio in the 3 rd Tier cities was also above the national average level. Especially lower loss ratio (around one fourth of national average) was achieved in the $5^{\text {th }}$ Tier cities instead of the 6th Tier cities. Dramatic loss of cropland was found in the higher-Tier cities, particularly the newly 1st, 2nd and the 1st Tier cities. For example, an average of around $340(337.94) \mathrm{km}^{2}$ cropland per city was permanently lost due to urbanization for each of the newly 1st cities. In contrast, less than 25 (23.91) $\mathrm{km}^{2}$ cropland loss per city experienced in the 5 th Tier cities (Table S3). The total area of lost cropland in the newly 1st Tier cities was around 3.6 times of the national average. The magnitude of high-quality cropland illustrated similar unbalanced pattern among the city hierarchy compared to the total cropland loss. For example, the lost area of high-quality cropland in the newly 1st Tier cities was also around three times of the national average.

The impact of urbanization on cropland were highly associated with the level of cities (Fig. 4, Table S2). Similar to the magnitudes of cropland losses (loss ratio, loss area), the pressure of urbanization on cropland indicated by the contribution rate also enhanced from the lower to higher Tier cities. For higher Tier cities such as the 1st Tier cities, urbanization resulted in direct cropland loss: over 80\% newly urban land was transformed from cropland loss. However, even for the lower-Tier cities (i.e. 4 th or lower), around $50 \%$ or more newly urban land occurred on cropland. Differed from the contribution rate, the high-quality ratio was higher in the 3rd and 4th Tier cities: over $40 \%$ cropland losses were of high-quality.

The impact of urbanization on cropland could also be revealed by the loss radius with larger loss ratios (Fig. 4). Higher Tier cities exhibited large loss radius. The loss radius with loss ratios around 10\% or more was $50 \mathrm{~km}$ for the 1st Tier cities, in contrast to less than $5 \mathrm{~km}$ in the 4 th or lower Tier cities. Additionally, loss ratios in higher-Tier 
a
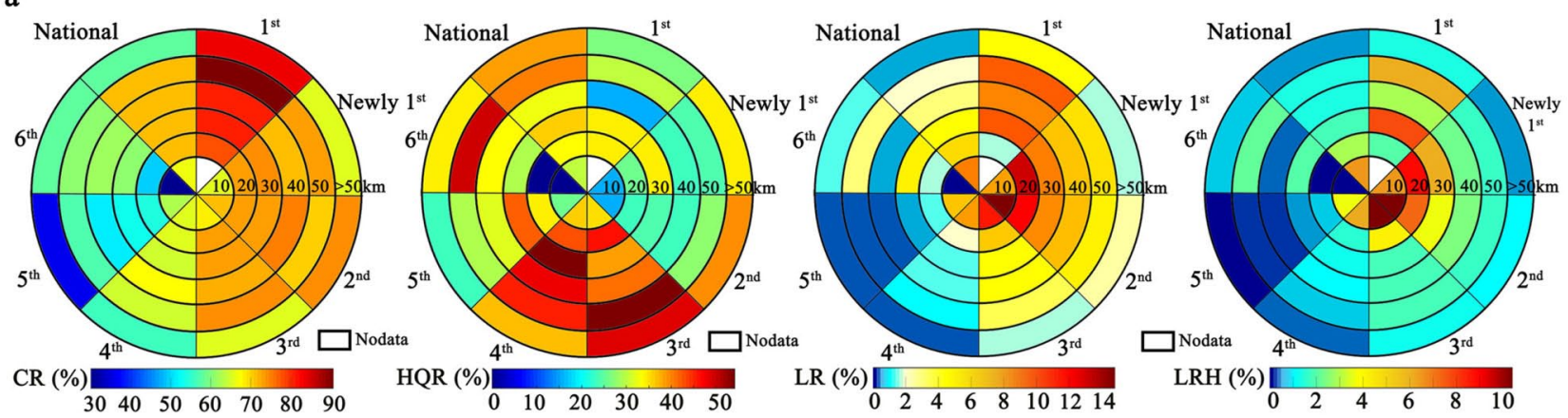

b
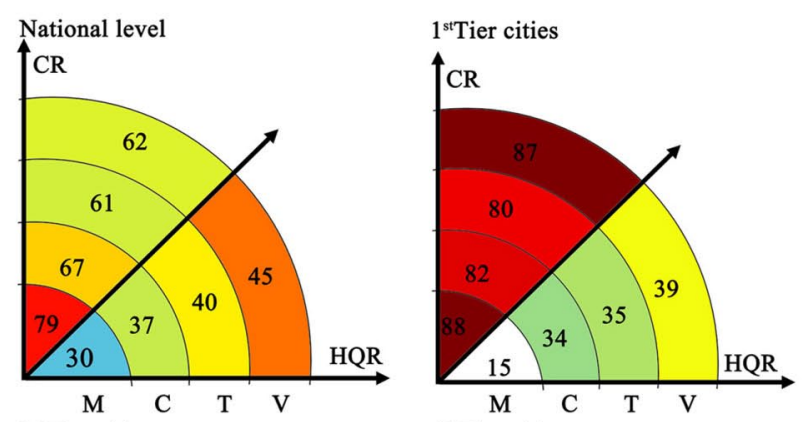

Newly $1^{\text {st }}$ Tier cities

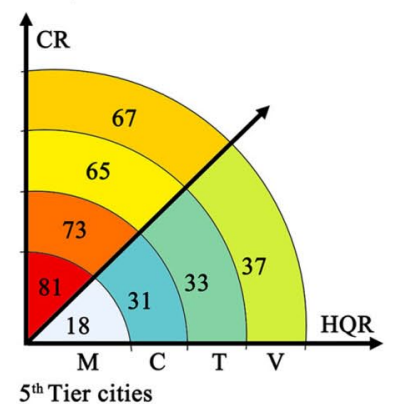

$2^{\text {nd }}$ Tier cities

$3^{\text {rd }}$ Tier cities

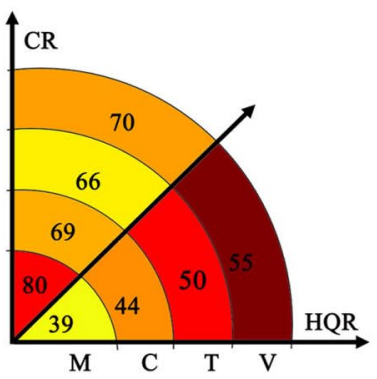

$4^{\text {th }}$ Tier cities
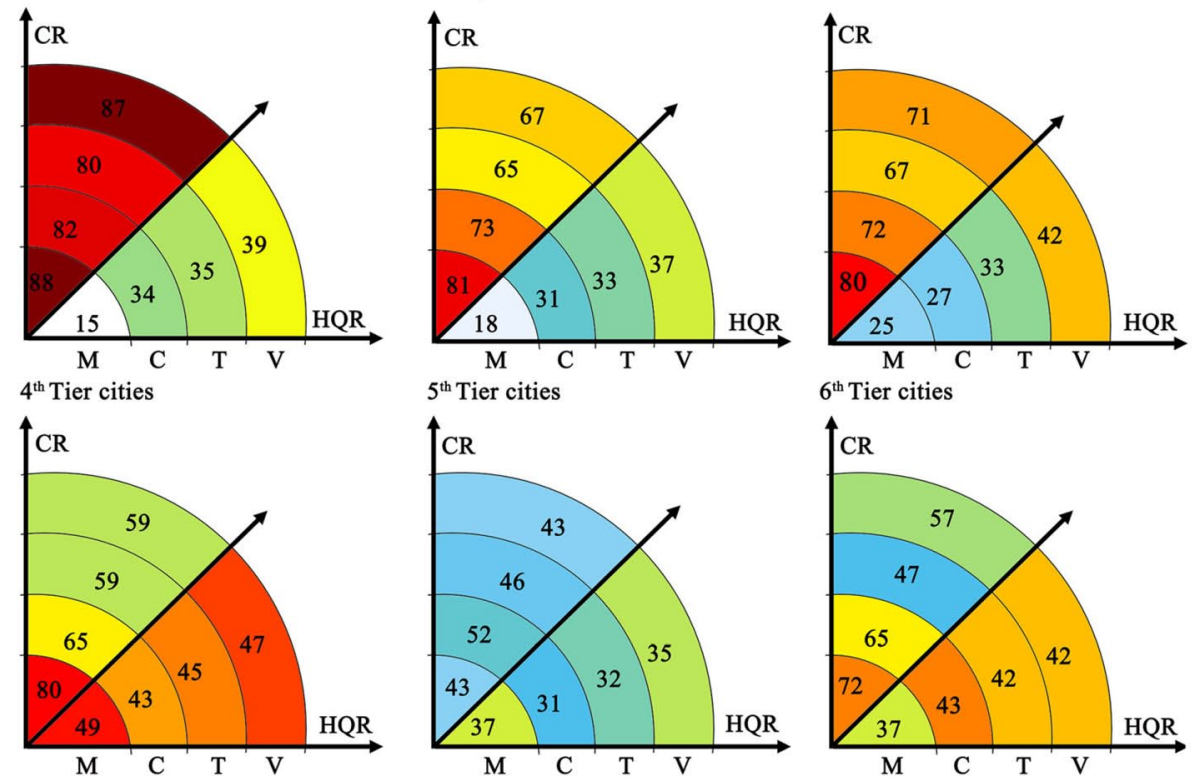

Urbanization mode

Urbanization mode

CR (\%)
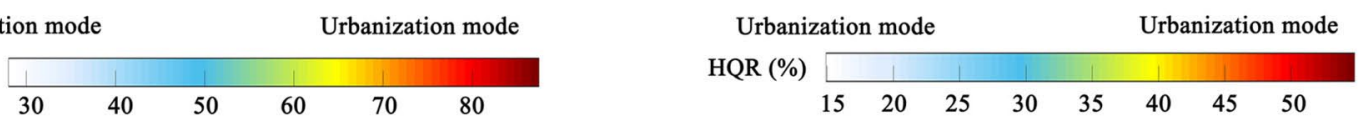

Fig. 4. Cropland losses among the city hierarchy associated with (a) distances to nearest city centers, (b) urbanization mode Patterns of losses with urbanization mode among the city hierarchy: a. contribution rate (CR), high-quality ratio (HQR); b. percentages of cropland due to different urbanization modes; c. percentages of high-quality cropland due to different urbanization modes.

cities declined more slowly with distances to cities. The pressure on cropland surrounding cities was very high: a majority ( $>60 \%$ ) of cropland losses located surrounding cities $(<40 \mathrm{~km})$ at national scale (Fig. S4). However, there were still one-quarter of national cropland losses occurred far from ( $>50 \mathrm{~km}$ ) any cities (6th or higher Tier cities) (Fig. S4). 
The asymmetric pattern of cropland losses could be summarized by the $\mathrm{Hu}$ line. Urbanization in the west of the $\mathrm{Hu}$ line were associated with less direct cropland loss (44\%) and high-quality ratio (21\%). Over 95\% losses in cropland (96\% loss in cropland and 97\% loss in high-quality cropland) located in the east of the Hu line. All these top 10 losses in cropland as well as high-quality cropland situated in the east surrounding the urban agglomeration such as the Yangtze River Delta, Jing-Jin-Ji and Central Henan (Fig. 3, Fig. S3). Most of the top 10 losses in cropland were from the higher Tier cities (the 2nd Tier or above). There were several outliers among the 3rd Tier cities such as the Taizhou and Yancheng in the Yangtze River Delta, which highlighted the significance of geographic location. A majority of top 10 losses in high-quality cropland were from the medium Tier cities (the 3rd and the 4th Tier).

\subsection{Cropland losses shaped by urbanization modes}

At national scale, city mode accounted for around two-fifths of national cropland losses. Each of town and mega-city mode was responsible for around one quarter of national cropland losses (Table S4). The village mode accounted for around one-eighth of national losses. However, cropland losses due to village mode urbanization enhanced gradually in recent few years (Table S4, Fig. S5).

At national scale, urbanization of mega-city mode resulted in more direct cropland losses (Fig. 4): one unit of newly urban land will introduce around 0.8 unit of cropland loss. For any of these four urbanization modes, over 6o\% newly urban land was converted from cropland. The town mode spared a little more cropland than other three modes. Differed from the contribution rate, the high-quality ratio increased gradually from mega-city, city and town modes to village mode. Specifically, nearly one half cropland losses due to village mode urbanization were of high-quality, in contrast less than one third due to megacity mode (Fig. 4).

Cropland losses shaped by urbanization modes were further analyzed for each Tier cities (Fig. 4). As regards to the contribution rate, the 1st and the 5th Tier cities illustrated distinct loss patterns associated with urbanization modes. In particularly, the 1st Tier cities resulted direct cropland losses for all urbanization modes (CR > 80\%). 
The 5th Tier cities achieved the lowest contribution rate from the mega-city modes instead of town mode. As regards to the high-quality ratio, the lower-Tier cities exhibited little variations with urbanization modes compared to higher Tier cities. The higher-Tier cities such as the 2nd Tier cities or above achieved much lower high-quality ratio (no more than 25\%) in mega-city mode than that in village mode. However, for the lower-Tier cities (4th or lower Tier cities), mega-city or city modes displayed slightly greater values in high quality ratio instead of the village modes. Specifically, the 4 th Tier cities were distinguished by a high-quality ratio of around 50\% in mega-city mode.

\subsection{Temporal patterns of cropland loss among the city hierarchy}

Diverse temporal patterns of losses emerged among different Tier cities (Fig. 5). The 1st Tier cities demonstrated continuous accelerated losses even in the 2010s. Losses in the newly 1st Tier cities per city were far more dramatic than those in other cities. The newly 1st Tier cities demonstrated more rapid acceleration followed by obvious deceleration processes with the turning point occurring 2 years

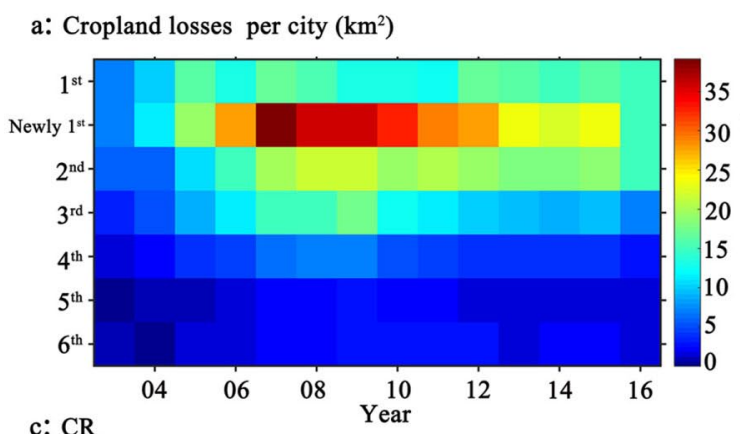

b: High-quality cropland losses per city $\left(\mathrm{km}^{2}\right)$
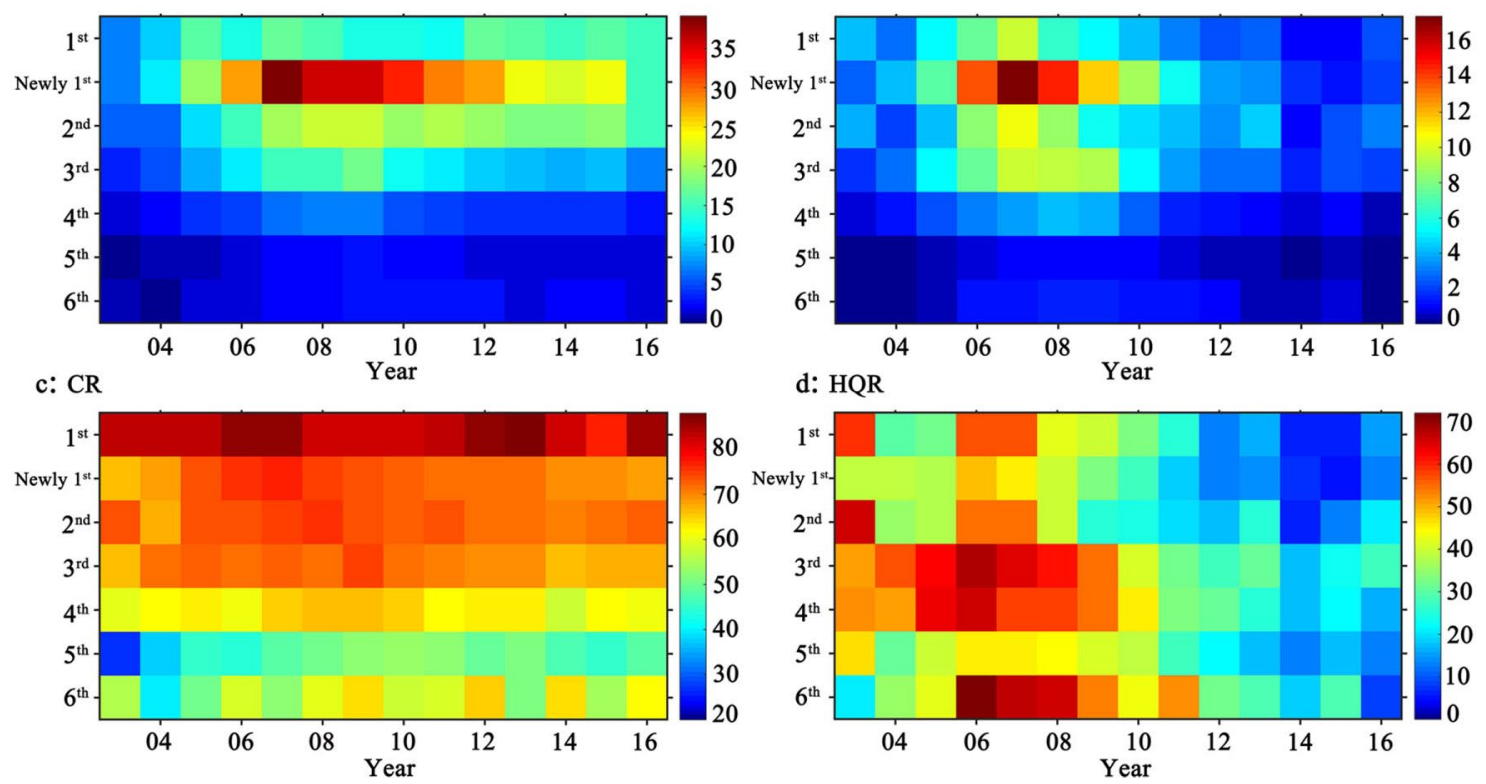

Fig. 5. Temporal patterns of annual losses of cropland per city, high-quality cropland per city, contribution rate (CR) and high-quality ratio (HQR) among different Tier cities. 
ahead of the national average. Compared to the newly 1st Tier cities, the 2nd or lower Tier cities experienced more continuous losses in the 2010s. Losses in the 5 th Tier cities were the lowest instead of the 6th Tier cities.

The 1st and newly 1st Tier cities achieved significant reductions of losses in high-quality cropland after 2007. The 2nd Tier cities ranked the highest in losses of high-quality cropland in the 2010s. Higher annual high-quality cropland loss was exhibited during the late 2000 for all Tier cities, with the peak values examined in 2007 (the 3rd Tier cities or above), 2008 (4th and 6th Tier cities) and 2009 (5th Tier cities).

Peak losses in all four urbanization modes emerged during the late 2000 (2007 for village mode, 2009 for others) (Table S4, Fig. S5). Losses due to mega-city and city modes continuously declined afterwards, however, losses in village mode accelerated in recent years. The contribution rate and high-quality ratio obtained the lowest values in 2015 and then slightly increased in recent years, especially in the village modes.

The contribution rate demonstrated much more stable temporal patterns compared to annual losses (Fig. 5). The high-quality ratio obviously declined, with peak values in 2003 (the 1st and 2nd Tier cities) or 2006 (others). The ranking of high-quality ratio changed annually: the top two were the 2nd and 1st Tier cities in 2003 but changed to the 3 rd and 4 th Tier cities afterwards.

\section{Discussion}

\subsection{Whether cropland loss was recently accelerated decoupled from urban expansion or not?}

Our answer is "yes, but only for high-quality cropland, and we should not be too optimistic". At a national scale, cropland has been increasingly strictly protected in recent years based on the following two findings. The first finding is the remarkable reduction in annual loss, which declined from $3500 \mathrm{~km}^{2}$ to around $1,700-2,200 \mathrm{~km}^{2}$ in recent years (Fig. 3). The most serious losses emerged in 2009 at a national scale, which is roughly coherence to a recent study based on 75 sample cities (Liu et al., 2019). The second finding is the more 
significant declines in both magnitudes and ratio of high-quality cropland loss. Annual high-quality losses decreased from around $1800 \mathrm{~km}^{2}$ in 2007 to $260-380 \mathrm{~km}^{2}$, and its ratio decreased from $60 \%$ to $12-20 \%$ in recent years (Table S3). High-quality cropland has been highly consumed by Beijing's urbanization, but this phenomenon has decreased over time (Song et al., 2015).

However, we should not be too optimistic due to the continuously high contribution rate of cropland in urbanization process. Losses in cropland were closely coupled with urban expansion in suburban as well as rural regions (Fig. 5) and would be continued in the coming 2020s. Therefore, there is an accelerated decoupling of the high-quality ratio instead of cropland losses from urban expansion. Reduction in cropland loss was largely attributed to the corresponding decline in newly urban land, which also peaked in 2009 and gradually decreased afterwards (Qiu et al., 2019). Therefore, cropland loss will continue to be affected by the continuing urbanization process (Deng et al., 2014). China's population urbanization rate in 2017 is $58.52 \%$ and is projected to reach 70\% in 2030 (FAO, 2013). Urbanization has become an important engine of China's modernization and will carry on in the coming future (Chen, 2007; Liu et al., 2014b). Land use competition for urbanization and food production has created a significant challenge in China and will continue in the coming few decades (Deng et al., 2014; Liu et al., 2019).

Competition for productive land will continue to be accelerated by the unbalance of high-quality cropland distribution and asymmetry of urbanization development processes in China (Chen, 2007; Seto and Ramankutty, 2016). Major agricultural regions have experienced rapid urbanization (Qiu et al., 2019). Although the high-quality ratio of losses at a national scale declined to $12 \%$ in 2014 , it gradually increased to $20 \%$ in 2016 . The high-quality ratio of losses in HuangHuai-Hai plain, the most productive region, has been continuously high $(40-42 \%)$ in recent years. Therefore, competition for high-quality cropland will continue along with the ongoing urbanization process of major megacities located in those productive regions (Qiu et al., 2019; Seto and Ramankutty, 2016). It is widely estimated that cropland contributes to more than half of newly urban land (Chen, 2007; Liu et al., 2019). This study found that the rate of cropland used for urban expansion at national scale was around $67 \%$, which is consistently 
much higher than 50\%. While one-fourth of total global cropland loss is projected to occur in China (Bren d'Amour et al., 2017), the findings of this study provide insightful evidences for decision makers to implement more adaptive cropland-protection policies in the future (Barthel et al., 2019).

\subsection{Has cropland loss been less severe in lower-Tiered cities and village mode?}

Cropland losses in lower-Tiered cities and their outlying were commonly assumed to be much less rigorous. Therefore, most of the previous research efforts concentrated on super-mega and mega cities based on sampling datasets instead of spatiotemporal continuous datasets across the city hierarchy (Liu et al., 2014a; Song and Liu, 2017; Zuo et al., 2018). This study revealed that urban expansion surrounding the medium-Tiered cities (e.g. 2nd, 3rd, 4th tiered cities) suffered most for high quality cropland losses. The past losses and potential trends for cropland losses in the medium-Tiered cities and lowerTiered cities should definitely not be neglected. Each Tiered cities exhibited their distinctive spatiotemporal patterns in cropland loss.

The 1st Tier cities ranked at the top considering their extremely high contribution rates (Fig. 4). Cropland losses in the 1st Tier cities were much more evenly distributed across space and time. The uniqueness of the 1st Tier cities reflects their corresponding ranking particularity in urban development patterns and national policies (Liu et al., 2014b; Tan et al., 2005). The newly 1st Tier cities had the most rigorous losses in cropland as well as of high-quality (Fig. 5). However, 2nd and 3rd Tier cities experienced more than 50\% of China's losses and might be responsible for more in the coming decades based on these trends. The 2nd Tier cities were responsible for the second largest proportion of losses at a national scale and ranked the top losses in high-quality cropland in the 2010s. The 2nd Tier cities showed no obvious deceleration in the 2010s and would probably suffer the most severe losses in the coming 2020s. The 3rd Tier cities accounted for the first largest proportion of losses at a national scale and ranked at the top in high-quality ratio (Fig. 4). The 3rd Tier cities demonstrated almost the same level of high-quality losses as that of the 2nd Tier cities. The 4th Tier cities should not be ignored, 
accounting for over one-sixth of total losses in high-quality cropland at national scale. The 4 th Tier cities, which are typically regional important cities, experienced much more losses than the lower cities (in both quantity and contribution rate). The high-quality ratio of losses in the 4th Tier cities was high and ranked as the top two (together with the 3 rd Tier cities) in the 2010s. The 5 th Tier cities showed the least rigorous losses. The 6th Tier cities exhibited more fluctuations in contribution rate compared to the others.

There was a close association between the ranking of cities and cropland losses as well as contribution rate at a national scale (Liu et al., 2019). It is true that more severe losses and higher contribution rates generally emerged in higher Tier cities. But the ranking of losses among the Tiered city system changed yearly, and there was an accelerated resequence for these higher-Tier cities in recent years. Higher-Tier cities experienced consistently higher contribution rates than lower-Tier cities (4th to 6th Tier cities), which contradicts a case study done in the 1990s (Tan et al., 2005). There was no significant difference in contribution rate among these higher Tier cities of the 3rd and above except the 1st Tier cities, which further confirms that rapid urbanization was experienced at the expense of cropland losses across moderate to mega cities (Kong, 2014; Liu et al., 2019). A recent study based on sampling datasets suggested that the acceleration stage of losses was sequenced by the ranking of cities (Liu et al., 2019). This study revealed that there was no significant difference in the acceleration stages among the Tiered city system in the first two decades of the 21st century, but the lower-Tier cities might be responsible for accelerated losses in cropland in the coming decades.

The patterns of losses with distance were highly shaped by the city hierarchy. At a national scale, the loss ratios declined from urban, suburban to rural regions, corresponding to the urban expansion patterns (Qiu et al., 2019). Higher-Tier cities had huge pressures upon their surrounding cropland in wider radiuses. Nevertheless, the cropland loss ratios adjacent to lower-Tier cities were also high despite smaller radiuses. The overlapping spatial distributions of urban expansion and the attributes of high-quality cropland determined the loss patterns with distance (Song et al., 2015). Acting on such leverage places to curb urban encroachment may navigate urban expansion away from fertile croplands (Barthel et al., 2019). 
This study found that cropland losses were highly shaped by unbalanced urbanization process, which could be characterized as the tiered system and urbanization mode in China. Higher Tier cities and mega-city urbanization modes resulted more direct losses of cropland, however, the medium Tier cities and village mode were more directly associated with high-quality cropland losses. The 5 th Tier cities and town urbanization mode achieved the least consumption of cropland, instead of the 6th Tier cities or village mode. This study enriched the location theory by considering the city Tier systems with urbanization modes. On the one hand, the Tier system accelerated cropland losses since losses (loss ratios) in the 2 nd or higher Tier cities were above twice or even over four times of the national average. On the other hand, dividing cities as Ties probably spared high-quality cropland since mega-city urbanization mode in the higher-Tier cities achieved much lower high-quality ratio, which could be accounted by the more strictly administration of mega-city urbanization mode in the higherTier cities as well as the corresponding geographic locations.

\subsection{Implications for cropland protection with consideration of urbanization mode}

Maintaining cultivated land is essential to food security, while the trend of urban expansion along with socioeconomic development is irreversible (Chen, 2007; Deng et al., 2014). China is making positive policy advances and is continuously moving towards strictly highquality cropland protection (Chen, 2007; Zuo et al., 2018). However, local governments are keen to land acquisition for urbanization and the cropland-protection policy might be degraded (Liu et al., 2014b; Skinner et al., 2001). Findings in this study called for target strategy for smart planning and efficient cropland protection with full considerations of city hierarchy and urbanization mode.

First, more frequent and strict monitoring should be strengthened in these hotspots of cropland losses. Monthly datasets of cropland should be developed for these hotspots (shown in Fig. 3) instead of annual update at national scale. High accuracy and frequency remote sensing mapping techniques are required for this purpose, especially the long-term time series classification approaches which could guaranteeing spatiotemporal continuity (Qiu et al., 2019; Wulder et al., 
2018). Strategies and policies to effectively steer patterns of urban expansion will be critical for preserving cropland (d'Amour et al., 2017; Song and Liu, 2017). We should devote unremitting efforts toward strictly suppressing the losses in high-quality cropland (Song and Liu, 2017).

Second, coordination and data sharing should be emphasized among government at different levels (central, provincial, municipal, county) encroaching both urban and rural regions. Overall coordinate urban and rural development is significant yet an arduous task which need to take the obvious regional discrepancies both in physical and socio-economic conditions into account (Long et al., 2010). High-quality cropland loss was consistently high in village mode urbanization. Encroach of high-quality cropland by village mode urbanization was difficult to be regulated probably due to the devolution of administrative responsibility in China and the increasing influence of powerful local economic interests (Skinner et al., 2001). Rural housing increased very fast in the past few decades but paid little attention to cherishing the scare land resources and lacked scientific village plan (Long et al., 2010).

Finally, possible adaptive planning and strategy was proposed. Cropland loss will proceed and will not be solved by suppressing urbanization (Deng et al., 2014). Rational and strategic land use planning aimed to relief unbalance distribution might be sought to mitigate the competition for good-quality cropland (Barthel et al., 2019; Cao et al., 2014; He et al., 2017). One is the relocation strategy of future urbanization to lower-Tier cities, given the less direct consumption of cropland in the western region. However, this relocation strategy might not be very realistic since efficient land use for non-agricultural economic development needs to be a priority in the eastern region (Long et al., 2010). Another strategy is the rational planning for optimized urbanization modes. This study found that the consumption of highquality cropland could definitely be under controlled, as evidenced from the mega-city mode urbanization in higher-Tier cities. It is worth to mention that the consumption of high-quality cropland by megacity mode urbanization has been very strictly controlled in China particularly in higher-Tier cities but not in lower-Tier cities. The policies designed to protect cultivated land by encouraging people move to cities may actually accelerate the occupation of cultivated land (Deng et 
al., 2015). Encouraging people to towns instead of living in small rural residential area (village mode) was recommended for the sake of treasuring cropland. Land area used for town mode urbanization is more economical and can play an important role in alleviating cropland loss (Deng et al., 2015).

A re-focusing of research including both national and local scales called for a more direct and possibly more efficient supervision on building new rural residential area (village mode), especially in medium-Tier cities. More attention should be paid to 2nd to 4 th Tier cities considering the challenges of suppressing the high-quality ratio and possible accelerating urbanization processes in the coming decades. Continuous work by government at all levels to reform land systems should be adopted considering regional discrepancies (Liu et al., 2018).

\section{Conclusions}

This study reported the asymmetric cropland losses shaped by the city hierarchy and urbanization mode using spatiotemporal statistic approaches supported by annual landscape changes datasets with great consistency and continuity.

The unbalanced urbanization strategy aggravated cropland losses regarding the extremely high contribution rate in the 1st Tier cities and consistently high contribution rate in higher-Tier cities. Cropland loss in medium or lower-Tier cities and even rural regions should definitely not be neglected given that they accounted for a majority of losses at national scale (total and of high-quality) and might be accelerated, particularly in high-quality percentages in the future. The impact of urbanization mode was closely associated with the city hierarchy. Mega-city mode urbanization occurred predominantly (80\%) at the expense of cropland, in contrast to prominent high-quality ratio in village/town mode. Ranking with urbanization mode was less obvious and even changed among the lower-Tier cities. Although an accelerated decline of high-quality ratio was achieved under strict protection, cropland losses due to village mode urbanization even intensified and nearly half were from high-quality, which deserved great attention. This study enriched the location theory by considering the city 
Tier systems with urbanization modes. Higher-Tier cities accelerated cropland losses but achieved lower high-quality ratio with mega-city urbanization mode. Findings in this study called for more adaptive planning and strict cropland protection under an integrated urbanrural land use policy framework.

\section{CRediT authorship contribution statement}

Bingwen Qiu: Conceptualization, Methodology, Writing - original draft, Writing review \& editing, Funding acquisition. Haiwen Li: Investigation, Formal analysis, Validation. Zhenghong Tang: Writing - review \& editing, Visualization. Chongcheng Chen: Formal analysis. Joe Berry: Writing - review \& editing, Supervision.

Acknowledgments This work was supported by the National Natural Science Foundation of China (grant no. 41771468, 41471362), funding from the Science Bureau of Fujian Province (2017Iooo8), the scholarship under the China Scholarship Council (CSC) (201806655006) and funding from Fuzhou University (GD1805). We are very grateful to the editors and anonymous reviewers for offering insightful suggestions and detailed comments which significantly improve the manuscript.

\section{Competing interest None.}

Appendix A. Supplementary data Supplementary material related to this article can be found attached to the article record in this archive.

\section{References}

Bai, X., Shi, P., Liu, Y., 2014. Society: realizing China's urban dream. Nature News 509, 158.

Barthel, S., Isendahl, C., Vis, B.N., Drescher, A., Evans, D.L., van Timmeren, A., 2019. Global urbanization and food production in direct competition for land: leverage places to mitigate impacts on SDG2 and on the Earth System. Anthr. Rev. 6, 71-97.

Cao, S., Lv, Y., Zheng, H., Wang, X., 2014. Challenges facing China's unbalanced urbanization strategy. Land Use Policy 39, 412-415. https://doi.org/10.1016/j. landusepol.2013.12.004.

Chen, J., 2007. Rapid urbanization in China: a real challenge to soil protection and food security. Catena 69, 1-15. https://doi.org/10.1016/j.catena.2006.04.019.

Chen, J., Chen, J., Liao, A., Cao, X., Chen, L., Chen, X., He, C., Han, G., Peng, S., Lu, M., Zhang, W., Tong, X., Mills, J., 2015. Global land cover mapping at $30 \mathrm{~m}$ resolution: a POK-based operational approach. Isprs J. Photogramm. Remote Sens. 103, 7-27. https://doi.org/10.1016/j.isprsjprs.2014.09.002. 
d'Amour, Bren, Reitsma, F., Baiocchi, G., Barthel, S., Güneralp, B., Erb, K.H., Haberl, H., Creutzig, F., Seto, K.C., 2017. Future urban land expansion and implications for global croplands. Proc. Natl. Acad. Sci. 114, 8939-8944. https://doi.org/10.1073/pnas.1606036114.

Deng, X., Lin, Y.L., Seto, K.C., 2014. Land-use competition between food production and Urban expansion in China. In: Seto, K.C., Reenberg, A. (Eds.), Rethinking Global Land Use in an Urban Era. The MIT Press.

Deng, X., Huang, J., Rozelle, S., Zhang, J., Li, Z., 2015. Impact of urbanization on cultivated land changes in China. Land Use Policy 45, 1-7. https://doi. org/10.1016/j.landusepol.2015.01.007.

Gaston, K.J., 2010. Urban Ecology. Oxford University Press.

He, C., Liu, Z., Xu, M., Ma, Q., Dou, Y., 2017. Urban expansion brought stress to food security in China: evidence from decreased cropland net primary productivity. Sci. Total Environ. 576, 660-670. https://doi.org/10.1016/j. scitotenv.2016.10.107.

Kong, X., 2014. China must protect high-quality arable land. Nature 506, 7.

Liu, Y., 2018. Introduction to land use and rural sustainability in China. Land Use Policy 74, 1-4. https://doi.org/10.1016/j.landusepol.2018.01.032.

Liu, Y., Li, Y., 2017. Revitalize the world's countryside. Nature News 548, 275.

Liu, J., Kuang, W., Zhang, Z., Xu, X., Qin, Y., Ning, J., Zhou, W., Zhang, S., Li, R., Yan, C., Wu, S., Shi, X., Jiang, N., Yu, D., Pan, X., Chi, W., 2014a. Spatiotemporal characteristics, patterns, and causes of land-use changes in China since the late 1980s. J. Geogr. Sci. 24, 195-210. https://doi.org/10.1007/s11442-014-1082-6.

Liu, Y., Fang, F., Li, Y., 2014b. Key issues of land use in China and implications for policy making. Land Use Policy 40, 6-12. https://doi.org/10.1016/j. landusepol.2013.03.013.

Liu, Y., Li, J., Yang, Y., 2018. Strategic adjustment of land use policy under the economic transformation. Land Use Policy 74, 5-14. https://doi.org/10.1016/j. landusepol.2017.07.005.

Liu, F., Zhang, Z., Zhao, X., Wang, X., Zuo, L., Wen, Q., Yi, L., Xu, J., Hu, S., Liu, B., 2019. Chinese cropland losses due to urban expansion in the past four decades. Sci. Total Environ. 650, 847-857. https://doi.org/10.1016/j. scitotenv.2018.09.091.

Long, H., Liu, Y., Wu, X., Dong, G., 2009. Spatio-temporal dynamic patterns of farmland and rural settlements in Su-xi-chang region: implications for building a new countryside in coastal China. Land Use Policy 26, 322-333. https://doi.org/10.1016/j.landusepol.2008.04.001.

Long, H., Liu, Y., Li, X., Chen, Y., 2010. Building new countryside in China: a geographical perspective. Land Use Policy 27, 457-470. https://doi. org/10.1016/j.landusepol.2009.06.006.

North, D.C., 1955. Location theory and regional economic growth. J. Polit. Econ. 63, 243-258. https://doi.org/10.1086/257668.

Ou, J., Liu, X., Wang, S., Xie, R., Li, X., 2019. Investigating the differentiated impacts of socioeconomic factors and urban forms on $\mathrm{CO} 2$ emissions: empirical 
evidence from Chinese cities of different developmental levels. J. Clean. Prod. 226, 601-614. https://doi.org/10.1016/j.jclepro.2019.04.123.

Qiu, B., Feng, M., Tang, Z., 2016. A simple smoother based on continuous wavelet transform: comparative evaluation based on the fidelity, smoothness and efficiency in phenological estimation. Int. J. Appl. Earth. Obs. 47, 91-101. https://doi.org/10.1016/j.jag.2015.11.009.

Qiu, B., Li, H., Chen, C., Tang, Z., Zhang, K., Berry, J., 2019. Tracking spatialtemporal landscape changes of impervious surface areas, bare lands, and inundation areas in China during 2001-2017. Land Degrad. Dev. https://doi. org/10.1002/ldr.3352. o.

Seto, K.C., Ramankutty, N., 2016. Hidden linkages between urbanization and food systems. Science 352, 943-945. https://doi.org/10.1126/science.aaf7439.

Skinner, M.W., Kuhn, R.G., Joseph, A.E., 2001. Agricultural land protection in China: a case study of local governance in Zhejiang Province. Land Use Policy 18, 329-340. https://doi.org/10.1016/S0264-8377(01)00026-6.

Skog, K.L., Steinnes, M., 2016. How do centrality, population growth and urban sprawl impact farmland conversion in Norway? Land Use Policy 59, 185-196. https://doi.org/10.1016/j.landusepol.2016.08.035.

Song, W., Liu, M., 2017. Farmland conversion decreases regional and national land quality in China. Land Degrad. Dev. 28, 459-471. https://doi.org/10.1002/ ldr.2518.

Song, W., Pijanowski, B.C., Tayyebi, A., 2015. Urban expansion and its consumption of high-quality farmland in Beijing, China. Ecol. Indic. 54, 60-70. https://doi.org/10.1016/j.ecolind.2015.02.015.

Song, X.P., Hansen, M.C., Stehman, S.V., Potapov, P.V., Tyukavina, A., Vermote, E.F., Townshend, J.R., 2018. Global land change from 1982 to 2016. Nature 56o, 639-643. https://doi.org/10.1038/s41586-018-0411-9.

Tan, M., Li, X., Xie, H., Lu, C., 2005. Urban land expansion and arable land loss in China-a case study of Beijing-tianjin-hebei region. Land Use Policy 22, 187196. https://doi.org/10.1016/j.landusepol.2004.03.003.

van Vliet, J., 2019. Direct and indirect loss of natural area from urban expansion. Nat. Sustain. https://doi.org/10.1038/s41893-019-0340-0.

van Vliet, J., Eitelberg, D.A., Verburg, P.H., 2017. A global analysis of land take in cropland areas and production displacement from urbanization. Glob. Environ. Chang.: A 43, 107-115. https://doi.org/10.1016/j.gloenvcha.2017.02.001.

Wulder, M.A., Coops, N.C., Roy, D.P., White, J.C., Hermosilla, T., 2018. Land cover 2.o. Int. J. Remote Sens. 39, 4254-4284. https://doi.org/10.1080/01431161.201 8.1452075.

Yan, H., Liu, J., Huang, H.Q., Tao, B., Cao, M., 2009. Assessing the consequence of land use change on agricultural productivity in China. Glob. Planet Change 67, 13-19. https://doi.org/10.1016/j.gloplacha.2008.12.012.

Zhang, Y., Xiao, X., Wu, X., Zhou, S., Zhang, G., Qin, Y., Dong, J., 2017. A global moderate resolution dataset of gross primary production of vegetation for 2000-2016. Sci. Data 4, 170165. https://doi.org/10.1038/sdata.2017.165. 
Zhang, S., Fang, C., Kuang, W., Sun, F., 2019. Comparison of changes in urban land Use/ Cover and efficiency of megaregions in China from 1980 to 2015. Remote Sens. (Basel) 11, 1834. https://doi.org/10.3390/rs11151834.

Zuo, L., Zhang, Z., Carlson, K.M., MacDonald, G.K., Brauman, K.A., Liu, Y., Zhang, W., Zhang, H., Wu, W., Zhao, X., Wang, X., Liu, B., Yi, L., Wen, Q., Liu, F., Xu, J., Hu, S., Sun, F., Gerber, J.S., West, P.C., 2018. Progress towards sustainable intensification in China challenged by land-use change. Nat. Sustain. 1, 304313. https://doi.org/10.1038/s41893-018-0076-2. 